\title{
Incidence of asthma and respiratory symptoms by sex, age and smoking in a community study
}

\author{
T.M.L. Eagan*, P.S. Bakke*, G.E. Eide", , A. Gulsvik*
}

Incidence of asthma and respiratory symptoms by sex, age and smoking in a community study. T.M.L. Eagan, P.S. Bakke, G.E. Eide, A. Gulsvik. (C) ERS Journals Ltd 2002. ABSTRACT: The purpose of this study was to establish incidence rates for a wide range of respiratory symptoms and asthma, and relate them to sex, age, and smoking habits.

A cohort established in 1985 as a random sample from the population of Western Norway, aged 15-70 yrs, was followed-up in 1996-1997. Of the initial cohort of 3,786 subjects, a total of 2,819 replied to mailed questionnaires at both baseline and follow-up.

The 11-yrs cumulative incidence of asthma was $4.0 \%$ in males and $3.5 \%$ in females. For respiratory symptoms, the cumulative incidences for both sexes varied between $\mathbf{2 . 0} \%$ (dyspnoea grade 4) and $\mathbf{2 5 . 8} \%$ (wheezing), being higher in females than males for most symptoms. For calculation of odds ratios (ORs) multivariate logistic regression analyses were used. The sex and smoking-adjusted incidences increased by age for all symptoms except wheezing and attacks of dyspnoea. Those starting to smoke within the follow-up had ORs of 1.9-2.2 for the cough symotoms compared to never-smokers, after adjusting for sex, age, and pack-yrs.

To conclude, the 11-yrs incidence of dyspnoea increased with increasing pack-yrs, after adjusting for sex, age, and changes in smoking habits. This indicated that when analysing other risk factors, adjustment has to be made for the risks posed by smoking, sex and age.

Eur Respir J 2002; 19: 599-605.
*Dept of Thoracic Medicine and ${ }^{\#}$ Centre for Clinical Research, Haukeland University Hospital, and Section for Medical Statistics, University of Bergen, Bergen, Norway.

Correspondence: T.M.L. Eagan, Dept of Thoracic Medicine, Haukeland University Hospital, N-5021 Bergen, Norway.

Fax: 4755975149

E-mail: Tomas.Eagan@med.uib.no or eagan@online.no

Keywords: Asthma cohort study

incidence

respiratory symptoms smoking

Received: May 232001

Accepted after revision November 16 2001
Community studies on the incidence of asthma in adults have mainly been based on self-reported information. The participants' access to health services, the diagnostic preferences of their doctors, and the recall of the participants may bias such studies. Compared to a diagnosis, respiratory symptoms are more unspecific, but are less subject to the biases discussed earlier. Various respiratory symptoms are predictors of hospitalization [1] and disability [2] from respiratory disease, as well as increased mortality [3].

Several community studies in North America [4-8] and Europe [9-15] have examined the incidence of respiratory symptoms and asthma. Most of the studies have examined smoking habits only at baseline. Only two $[4,13]$ of the studies have examined the relationship of changes in smoking habits during the follow-up to the incidence of respiratory symptoms. Most of the studies have included less than three respiratory symptoms $[6,8-10,12,15]$ and several of the studies are restricted to specific groups i.e. sex $[8,9]$ or age $[8,14,15]$. No population-based data, on several respiratory symptoms in a wide age span of the population, is available from Northern Europe. None of the studies cited earlier have examined the incidence of various degrees of breathlessness in males and females separately.

The objective of the present study was to assess the 11-yrs incidence of asthma and respiratory symptoms in a North-European general population. How these incidences varied by sex, age and smoking was examined also.

\section{Methods}

\section{Subjects and study design}

The baseline study was conducted in 1985 , and the details of the data collection have been described previously [16]. In the county of Hordaland, Western Norway, $1.8 \%$ of the population aged $15-70$ yrs were randomly sampled and sent a questionnaire regarding respiratory symptoms, disease, smoking, and occupational airborne exposures. Of these initial 4,992 subjects, 3,786 lived in the city of Bergen or the 11 surrounding municipalities. This subsample of 3,786 subjects was the basis for a follow-up in 1996/1997, where the subjects were invited to an examination including lung function testing. Included in the invitation was a questionnaire. This follow-up questionnaire was similar to the questionnaire in 1985 regarding respiratory symptoms, asthma, and smoking, but added some questions relating to education and family history. Of the initial 3,786 subjects in 1985 , a total of $3,370(89.0 \%)$ replied to the initial 
questionnaire or one of the two reminders. A total of 189 subjects died between 1985-1996, leaving 3,181 subjects eligible for follow-up. A total of $2,819(88.6 \%)$ returned the questionnaire after two reminders.

\section{The questionnaires}

The exact wording of the questions on all respiratory symptoms and asthma is given in the Appendix. The questions on respiratory symptoms and asthma have been validated previously against lung function and bronchial reactivity [17] and compared with the British Medical Research Council questionnaire on chronic bronchitis [18]. Changes in smoking habits were defined by whether they were never-, ex-, or current smokers at baseline and follow-up, and recoded into five categories: never-to-never, non-tocurrent, current-to-current, current-to-ex, or ex-to-ex. The non-to-current group consisted of both neverto-current and ex-to-current smokers. Subjects giving inconsistent replies, like current-to-never, were excluded from the analyses. One pack-yr was defined as 20 cigarettes $\cdot \mathrm{day}^{-1} \cdot \mathrm{yr}^{-1}$. For the pipe smokers, 1 pack-yr was defined as having smoked $50 \mathrm{~g}$ of tobacco $\cdot$ week $^{-1} \cdot \mathrm{yr}^{-1}$. The information obtained by the questions on smoking habits has been validated previously against thiocyanate [19]. The cumulative incidence was defined as the number of new cases at follow-up having a symptom or asthma, divided by the subjects at risk of becoming symptomatic or asthmatic, that is those not having the symptom in question or asthma at baseline [20]. In this study, the term cumulative incidence to yearly incidence was used for two reasons: Firstly, the data collection at baseline lasted 2 months. At follow-up it lasted 8 months due to the inclusion of a clinical examination. Thus, some subjects had a follow-up period that was 6 months longer than others. Secondly, it is not known at what time the incident event occurred within the $11 \mathrm{yrs}$ of follow-up.

\section{Statistical analysis}

Logistic regression, with the likelihood ratio test, was used to estimate the adjusted odds ratio (OR) and $95 \%$ confidence interval. The incidence of the symptom in question or asthma was the dependent variable, and sex, age, changes in smoking habits, and pack-yrs were independent variables. Increasing pack-yrs showed a linear relationship to the incidences of the symptoms and asthma, and was used as a continuous variable. For age, the relationship was more complicated, with a marked increase in incidence after the age of 50. Thus, age was modelled as a grouped variable with three categories. All firstorder interactions between the independent variables were examined, amounting to a total of 66 separate regression analyses. A p-value of 0.01 was considered significant for the interactions, to reduce the possibility of a type- 1 error. Otherwise, a $\mathrm{p}=0.05$ was used.

When calculating the incidence of, for example, dyspnoea grade 3 , both those being asymptomatic and those having dyspnoea grade 1 or 2 at baseline were incident cases. Thus, the incidence of dyspnoea grade 2-4 did not take into account the magnitude of the increase. To examine this aspect, a log-linear regression model with a three categories outcome variable was used. The three categories of outcome were an increase in dyspnoea of 1,2 , or more grades. The variable changes in smoking habits were not significant predictors in the model, and were excluded, leaving sex, age, and pack-yrs as independent variables.

\section{Results}

The characteristics of the study population are given in table 1 . There were slightly more females in the oldest age group than males. More females were never-to-never smokers, whereas a larger number of males were current-to-ex and ex-to-ex smokers. Amongst current or exsmokers at follow-up, mean \pm SD pack-yrs were $17.1 \pm 14$.6 for males and $12.1 \pm 9.8$ for females.

Within the study population, there was a marked increase in the prevalence of all symptoms and asthma between baseline and follow-up (table 2). This increase was lowest for dyspnoea grade 4 and highest for wheezing. For all symptoms the increase in prevalence was higher in females than males, in most cases by a factor of $>2$. This difference in increased prevalence between sexes reached statistical significance for all symptoms except phlegm cough, dyspnoea grade 1, and attacks of dyspnoea.

The cumulative incidences for asthma and the 10 respiratory symptoms are given in table 3 . The incidences of asthma and phlegm cough were slightly higher in males. Daytime cough had similar incidences

Table 1. - Characteristics of the Hordaland follow-up study cohort

\begin{tabular}{lcc}
\hline & Males & Females \\
\hline Total & $1352(48.0)$ & $1467(52.0)$ \\
Age in 1985 yrs & & \\
15-29 & $489(36.2)$ & $491(33.5)$ \\
$30-49$ & $541(40.0)$ & $573(39.1)$ \\
50-70 & $322(23.8)$ & $403(27.5)$ \\
Changes in smoking habits & & \\
$\quad$ Never-to-never & $427(32.9)$ & $631(45.6)$ \\
Non-to-current & $67(5.2)$ & $68(4.9)$ \\
Current-to-current & $395(30.4)$ & $381(27.5)$ \\
Current-to-ex & $157(12.1)$ & $136(9.8)$ \\
Ex-to-ex & $253(19.5)$ & $167(12.1)$ \\
Pack-yrs & & \\
0 & $427(32.9)$ & $631(45.3)$ \\
1-9 & $312(24.1)$ & $392(28.1)$ \\
10-19 & $270(20.8)$ & $226(16.2)$ \\
$20+$ & $287(22.1)$ & $144(10.3)$ \\
\hline
\end{tabular}

Data are presented as $\mathrm{n}(\%){ }^{*}$ : some subjects were not included in these categories due to lack of reporting of smoking, or inconsistencies; ": not all subjects responded to questions about the number of cigarettes they smoked per day or the number of years they had smoked. 
Table 2. - Prevalence of respiratory symptoms and asthma at baseline and follow-up

\begin{tabular}{|c|c|c|c|c|c|c|c|c|c|}
\hline & \multicolumn{3}{|c|}{ Males $\#$} & \multicolumn{3}{|c|}{ Females } & \multicolumn{3}{|c|}{ All subjects ${ }^{+}$} \\
\hline & 1985 & $1996 / 1997$ & Increase & 1985 & $1996 / 1997$ & Increase & 1985 & $1996 / 1997$ & Increase \\
\hline \multicolumn{10}{|l|}{$\begin{array}{l}\text { Respiratory symptoms } \\
\text { and asthma }\end{array}$} \\
\hline Asthma & 3.6 & 6.4 & 2.8 & 2.9 & 5.6 & 2.7 & 3.4 & 6.0 & 2.6 \\
\hline Morning cough & 23.7 & 24.7 & 1.0 & 18.2 & 25.0 & 6.8 & 21.1 & 24.9 & 3.8 \\
\hline Daytime cough & 13.4 & 15.5 & 2.1 & 9.1 & 14.7 & 5.6 & 11.9 & 15.1 & 3.2 \\
\hline Chronic cough & 11.5 & 12.1 & 0.6 & 8.0 & 12.3 & 4.3 & 10.0 & 12.2 & 2.2 \\
\hline Phlegm cough & 25.0 & 26.3 & 1.3 & 17.2 & 21.1 & 3.9 & 20.7 & 23.6 & 2.9 \\
\hline Dyspnoea grade 1 & 15.2 & 18.9 & 3.7 & 20.4 & 25.2 & 4.8 & 19.1 & 22.2 & 3.1 \\
\hline Dyspnoea grade 2 & 8.1 & 12.1 & 4.0 & 11.8 & 20.1 & 8.3 & 11.2 & 16.2 & 5.0 \\
\hline Dyspnoea grade 3 & 2.1 & 4.4 & 2.3 & 2.3 & 6.5 & 4.2 & 2.9 & 5.5 & 2.6 \\
\hline Dyspnoea grade 4 & 1.0 & 1.6 & 0.6 & 1.0 & 2.9 & 1.9 & 1.4 & 2.3 & 0.9 \\
\hline Attacks of dyspnoea & 11.3 & 12.7 & 1.4 & 13.0 & 15.9 & 2.9 & 12.2 & 14.4 & 2.2 \\
\hline Wheezing & 22.0 & 35.7 & 13.7 & 18.5 & 36.5 & 18.0 & 20.6 & 36.1 & 15.5 \\
\hline
\end{tabular}

Data are presented as $\%{ }^{\#}: \mathrm{n}=1,352 ;{ }^{\uparrow}: \mathrm{n}=1,467 ;{ }^{+}: \mathrm{n}=2,819$.

in both sexes, whereas all the other symptoms showed a higher incidence in females, by a factor of 1.2-1.9. The incidences of attacks of dyspnoea and wheezing were highest in the youngest age group. For the other symptoms and asthma, the incidences were highest in the oldest age group. The incidence of all grades of dyspnoea were 2-3 times higher in the oldest age group, than in the other age groups. Current smokers at follow-up (i.e. non-to-current and current-tocurrent smokers) had 1.5-2 times higher incidences of wheezing and cough than never-to-never smokers. The current-to-ex group had the highest incidence of asthma and dyspnoea grade 2-4. The incidence of asthma and all symptoms, except dyspnoea grade 4, increased with increasing number of pack-yrs.

After adjusting for age, changes in smoking habits, and pack-yrs, the incidence for the dyspnoea symptoms and wheezing were higher in females than males (table 4). The incidence of asthma and all symptoms, except wheezing and attacks of dyspnoea, were increased in those aged $>50$ yrs after adjusting for sex and smoking habits. The non-to-current and current-to-current smokers had an increased incidence of the cough symptoms, even after adjusting for pack-yrs, sex, and age. Pack-yrs smoked was a risk factor for increased incidence of all symptoms and asthma, including the cough symptoms, after adjustment for changes in smoking habits, sex, and age.

Of the 66 interaction analyses, only three reached statistical significance. Those were the interactions between age and pack-yrs and age and changes in smoking habits for morning cough, and between age and changes in smoking habits for dyspnoea grade 1 . With higher age, the effect of pack-yrs diminished. OR per 10 pack-yrs increase in smoking for the incidence of morning cough was 2.2 in those aged 15-29 yrs, 1.4 in those aged 3-49 yrs, and 1.0 in those aged $>50$ yrs. In the interaction between age and changes in smoking habits, the adjusted ORs for dyspnoea grade 1 in current-to-current smokers, as compared to never-to-never smokers, was 2.3 in the age group 15-29 yrs, 1.4 in the age group 30-49 yrs, and 0.6 in those aged $50-70$ yrs. The corresponding figures for morning cough were 2.4, 1.9, and 0.7, respectively.

Females had higher ORs for the incidence of dyspnoea regardless of the magnitude of the increase (table 5). In those aged $>50$ yrs, the OR for a small $(\Delta 1)$ increase in dyspnoea was 1.4 , for a modest increase $(\Delta 2) 2.1$, and for a large increase $(\Delta 3-4) 2.4$. Pack-yrs were significant predictors for all degrees of increase of dyspnoea, with no apparent trend between varying degrees of increases.

\section{Discussion}

The 11-yrs cumulative incidence for asthma was $3.7 \%$. For the other respiratory symptoms the corresponding figures varied between 2.0\% (dyspnoea grade 4 ) and $25.8 \%$ (wheezing). Being female was an independent predictor for increased incidence of all symptoms except daytime cough and phlegm when coughing, while no difference was observed for the incidences of asthma. The sex- and smoking-adjusted incidences increased by age for all the symptoms except for attacks of dyspnoea and wheezing. Nonto-current and current-to-current smoking tended to have higher incidences of the cough symptoms as compared to never-to-never smokers, while such a trend was not found for the dyspnoea symptoms.

There are some methodological aspects to consider. Arguably, the leading cause of bias in a cohort study from a general population is loss to follow-up. Both at baseline and at follow-up the response rate was high, at $89 \%$. A total of $81 \%$ of the initial sample replied to both questionnaires. Further, the baseline sample was representative to the study population with respect to the distribution of sex, age, smoking habits, and respiratory symptoms [16]. Finally, the authors analysed whether inclusion of only initial responders or all responders at follow-up affected the relationship between sex, age, and changes in smoking habits and the incidence of the respiratory symptoms [21]. No changes in the incidences were found. Prior studies 


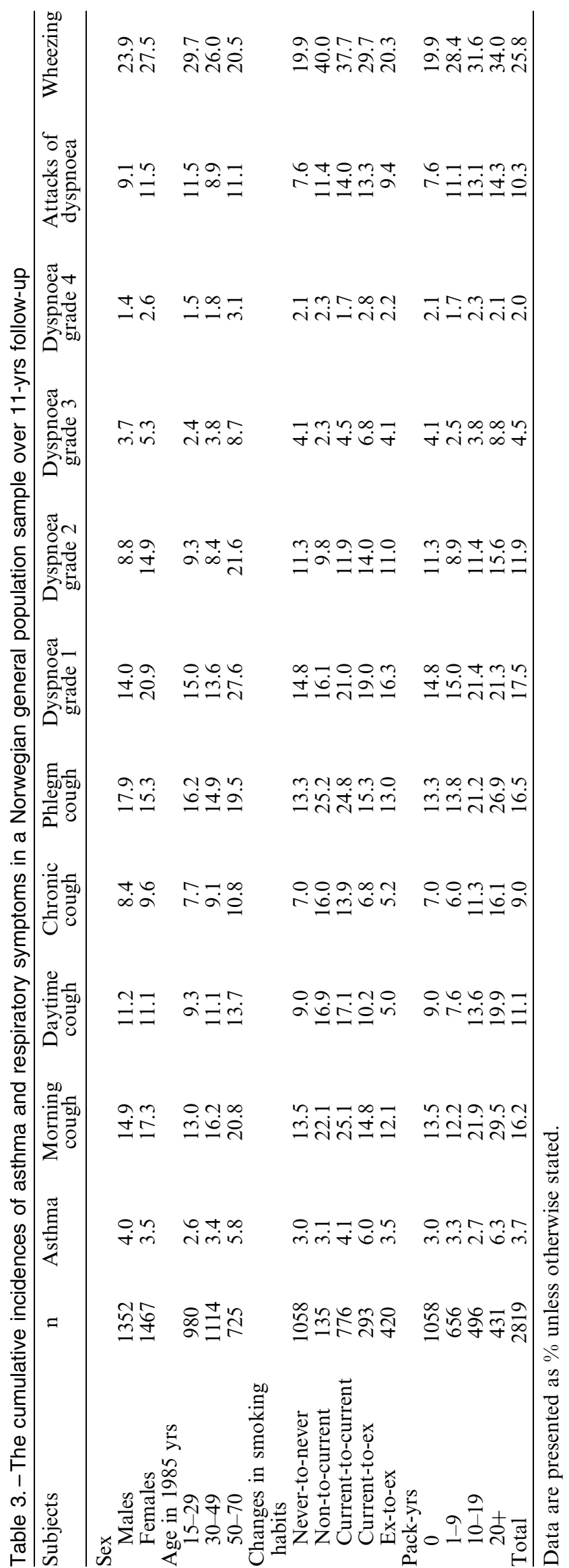

have analysed the smoking exposure differently. The most common model is by dividing the smokers at baseline into never-, ex-, and current smokers [7, 1012]. Two studies have examined the relationship between changes in smoking habits and the incidence of symptoms, thus categorizing smokers as never-tonever, current-to-ex etc. $[4,13]$. The advantage of this latter approach is to enable an analysis of differences in incidence amongst those starting to smoke within the follow-up period. However, with only two observational points in the cohort study, the incident event could theoretically precede this change in smoking habit, a problem avoided with the baseline approach. The problem with the baseline-only approach to the smoking exposure is that it could have underestimated the effect of smoking. A never-to-current smoker is likely to have a higher risk than a never-to-never smoker, however, both are classified as never-smokers at baseline.

Although several studies have noted a recent rise in asthma prevalence [22-25], a convincing rise in asthma incidence has not been found. Previous cohort studies on asthma incidence $[5,6,8,10,12,13,15]$ have reported annual figures varying from $0.10 \%$ [13] to $0.49 \%$ [12] in males, and from $0.17 \%$ [15] to $0.52 \%$ [12] in females. The findings in this study indicate a yearly incidence of $0.36 \%$ for males, and $0.32 \%$ for females. The studies cited were conducted from 1959-1996. These studies revealed no trends towards a higher or lower incidence. Theoretically, an increasing prevalence, and a stable incidence could imply that the remission rate is decreasing. However, the interpretation should be cautious as the incidence studies of asthma differ in diagnostic criteria of the disease, as well as composition of the study population.

Of the previous studies on incidence of symptoms, two have found higher incidences of dyspnoea in females compared to males [4, 13]. One of these studies found a higher incidence of cough symptoms in males compared to females [13]. In the study by KrzYZANOWSKI et al. [13], females in Tucson had higher incidences of asthma than males, whereas in Cracow no difference between sexes was found. Other studies have found similar incidences of asthma in males and females [12], age-specific sex differences [6], or a higher asthma incidence in females [15].

In the present study, females had higher incidences of all the respiratory symptoms, except for phlegm when coughing. Furthermore, it was observed that the magnitude of the increase in dyspnoea was higher in females than in males after adjusting for age and smoking habits (table 5). Previous studies have shown that females tend to under-report phlegm when coughing [26]. If this were the case in the present cohort, it would mean that although the crude incidences of all symptoms were higher among females, the crude incidence of asthma was higher among males ( $4.0 \%$ versus $3.5 \%$, table 3 ). This lack of correlation between symptoms and asthma between sexes can in principle have two different explanations. First, symptoms in males and females could be inherently different, representing either a difference in the underlying disease mechanism, or a difference in the perception of the symptom. In a 


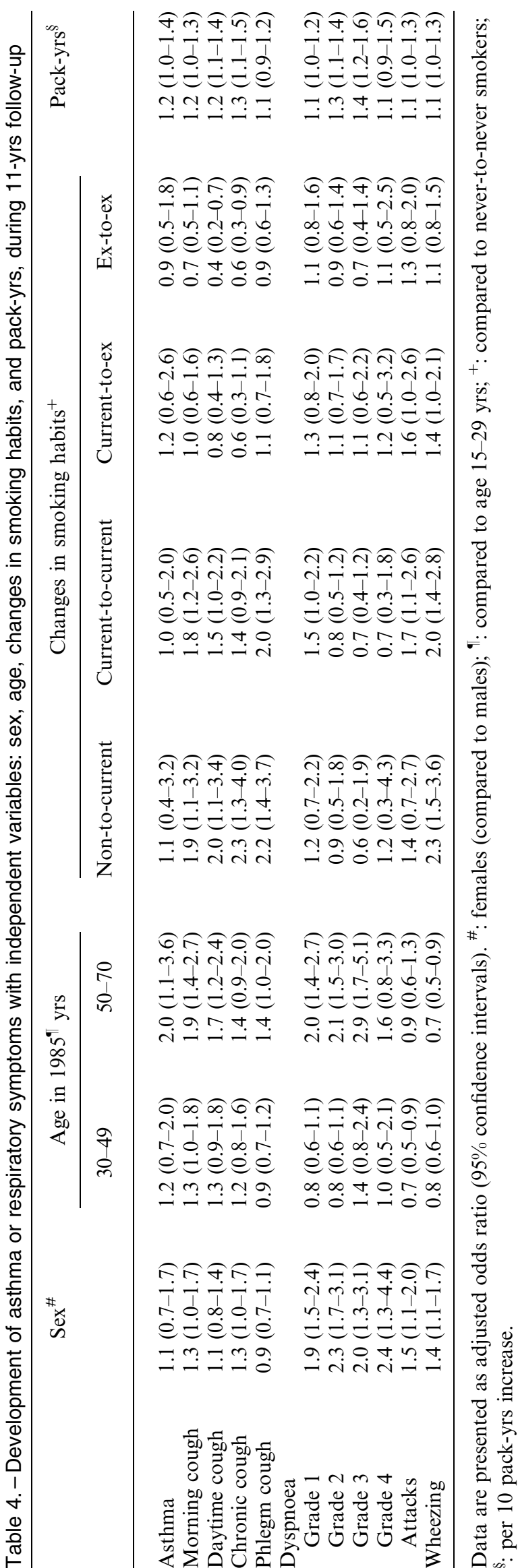

study by KAUFFMANN et al. [27], females were found to report more shortness of breath than males for any given forced expiratory volume in one second deficit. Females have been found also to have a more sensitive cough reflex, given an equal cough stimulant [28, 29]. Furthermore, it has been argued that females are more perceptive to breathlessness than males, due to cyclical hormonal variations [26]. However, this cannot explain the sex difference in dyspnoea found at ages $>50 \mathrm{yrs}$. Second, asthma could be underdiagnosed in females, or overdiagnosed in males.

Age $>50$ yrs was an independent predictor of the incidence of asthma, all the cough symptoms, and all degrees of dyspnoea. This could have been due to cumulative exposure to factors other than smoking, like dust or gas, exposure in the workplace. With increasing age, an increase in comorbidity of other diseases, like heart disease causing dyspnoea, and gastro-esophageal reflux disease causing cough, would be expected. Finally, the age trend could reflect a cohort effect. Those in the oldest age group might have experienced exposures in early life to a higher extent than today's younger age group. Those exposures might cause symptoms in later life.

Similar trends in the incidence of asthma, and the cough and dyspnoea symptoms have been observed by other studies $[6,12,13]$, although in the Tucson study [13] there was no age trend for the incidence of chronic phlegm. Differences in levels of air pollution and occupational airborne exposure between the studies may partly explain these contradictory findings. However, differences in wording of the questions could also influence the results.

The authors were unable to find any previous studies examining predictors of varying degrees of increase in dyspnoea. In the log-linear analysis, sex, age, and, pack-yrs were significant predictors. However, only in those aged $>50$ yrs was there an increase in the ORs for increasing degrees of dyspnoea. This shows that the eldest population is at higher risk both for developing dyspnoea as well as the more severe degrees of dyspnoea.

The effect of smoking on the incidences between the cough and dyspnoea symptoms differed. Regarding the cough symptoms, the current-to-current and non-to-current smokers had higher adjusted ORs, and the ex-to-ex and current-to-exsmokers lower ORs compared to the never-to-never smokers. Regarding the dyspnoea symptoms, no overt differences in adjusted ORs were noted by changes in smoking habits (table 4). Number of pack-yrs was a risk factor for all the symptoms. This suggests that the development of dyspnoea is dependent on the total smoking consumption, and reflects irreversible airway obstruction and obliteration of coronary vessels. For the cough symptoms the effect of smoking is more immediate through airway inflammation, phlegm production, and mucociliary transport, all improving on smoking cessation.

The results from this study regarding the effects of smoking habits on the cough symptoms are in agreement with those of the only other two papers presenting data on incidence of symptoms by change 
Table 5. - Increase in dyspnoea 1, 2, or 3-4 grades by independent variables sex, age, and pack-yrs in a multinominal logistic regression model

$\Delta 1$ increase $\Delta 2$ increase $\Delta 3-4$ increase

Compared to no increase in dyspnoea

\begin{tabular}{|c|c|c|c|}
\hline \multicolumn{4}{|l|}{ Sex } \\
\hline Females (compared to males) & $1.6(1.2-2.2)$ & $2.4(1.7-3.4)$ & $1.8(1.0-3.1)$ \\
\hline \multicolumn{4}{|l|}{ Age in 1985 (compared to $15-29)$ yrs } \\
\hline $30-49$ & $0.9(0.6-1.2)$ & $0.8(0.5-1.2)$ & $1.0(0.5-2.0)$ \\
\hline $50-70$ & $1.4(0.9-2.0)$ & $2.1(1.5-3.0)$ & $2.4(1.3-4.6)$ \\
\hline \multicolumn{4}{|l|}{ Pack-yrs } \\
\hline Per 10 pack-yrs increase & $1.3(1.1-1.4)$ & $1.1(1.0-1.3)$ & $1.2(1.0-1.5)$ \\
\hline
\end{tabular}

Data are presented as odds ratio ( $95 \%$ confidence interval).

in smoking habits [4, 13]. None of these studies covered Western European populations.

The effect of smoking on the incidence of morning cough and dyspnoea grade 1 decreased with increasing age. With ageing, the effect of degenerative changes and comorbidity on the incidence is likely to increase, reducing the relative importance of smoking. Of the studies that have examined the relationship of smoking to asthma incidence some have observed that smokers had a higher incidence of asthma [12], some that there was no relationship $[6,10,15]$ and some that smokers had a lower incidence [8]. The different findings could reflect that the effect of smoking on asthma incidence is weak. Different diagnostic criteria between the studies warrant a cautious interpretation. It is worth noting that none of the studies examined asthma incidence by change in smoking habits, but rather by smoking status at baseline. This study gives cumulative incidence rates for a wide range of respiratory symptoms and asthma. Smoking, either measured by changes in smoking habits, or as pack-yrs, is a clear risk factor for all the respiratory symptoms and asthma.

Possible other risk factors for the incidence of respiratory symptoms and asthma include heredity, working exposures, air pollution, and socioeconomic status. When analysing these other possible risk factors it is important to adjust for the risk posed by smoking, as well as by sex, and age.

\section{Appendix}

The wording of the questions in 1985 and 1996/1997 regarding respiratory symptoms and asthma were identical:

Do you usually cough or clear your throat in the morning? (yes, no).

Do you usually cough during the rest of the day? (yes, no).

Do you usually have phlegm when coughing? (yes, no).

Do you have a cough for three months or more altogether during a year? (yes, no).

Are you more breathless than other people of your own age when walking uphill? (yes, no) (dyspnoea, grade 1).
Are you breathless when you climb two flights of stairs at an ordinary pace? (yes, no) (dyspnoea grade 2).

Are you breathless walking at a normal pace on level ground? (yes, no) (dyspnoea grade 3).

Are you breathless while at rest? (yes, no) (dyspnoea grade 4).

Do you sometimes experience attacks of breathlessness? (yes, no).

Do you ever have wheezing in your chest? (yes, no).

Have you ever been treated by a doctor or been hospitalized for asthma? (yes, no, do not know).

Have you ever been treated by a physician, or have you been hospitalized for asthma? (yes, no).

\section{References}

1. Vestbo J, Rasmussen FV. Respiratory symptoms and FEV1 as predictors of hospitalization and medication in the following 12 years due to respiratory disease. Eur Respir J 1989; 2: 710-715.

2. Comstock GW, Stone RW, Tonascia JA, Johnson $\mathrm{DH}$. Respiratory survey findings as predictors of disability from respiratory diseases. Am Rev Respir Dis 1981; 124: 367-371.

3. Lange P, Nyboe J, Appleyard M, Jensen G, Schnohr P. Ventilatory function and chronic mucus hypersecretion as predictors of death from lung cancer. Am Rev Respir Dis 1990; 141: 613-617.

4. Beck GJ, Doyle CA, Schachter EN. A longitudinal study of respiratory health in a rural community. $\mathrm{Am}$ Rev Respir Dis 1982; 125: 375-381.

5. Broder I, Higgins MW, Mathews KP, Keller JB. Epidemiology of asthma and allergic rhinitis in a total community, Tecumseh, Michigan. IV. Natural history. J Allergy Clin Immunol 1974; 54: 100-110.

6. Dodge RR, Burrows B. The prevalence and incidence of asthma and asthma-like symptoms in a general population sample. Am Rev Respir Dis 1980; 122: 567575.

7. Ferris BG, Higgins IT, Higgins MW, Peters JM. Chronic nonspecific respiratory disease in Berlin, New Hampshire, 1961 to 1967. A follow-up study. Am Rev Respir Dis 1973; 107: 110-122.

8. Troisi RJ, Speizer FE, Rosner B, Trichopoulos D, Willett WC. Cigarette smoking and incidence of chronic bronchitis and asthma in women. Chest 1995; 108: 1557-1561. 
9. Vestbo J, Knudsen KM, Rasmussen FV. The effect of smoking and occupation on changes in respiratory symptoms in middle-aged Danish men. Eur Respir $J$ 1990; 3: 880-885.

10. Vesterinen E, Kaprio J, Koskenvuo M. Prospective study of asthma in relation to smoking habits among 14,729 adults. Thorax 1988; 43: 534-539.

11. Xu X, Rijcken B, Schouten JP, Weiss ST. Airways responsiveness and development and remission of chronic respiratory symptoms in adults. Lancet 1997; 350: 1431-1434.

12. Ronmark E, Lundback B, Jonsson E, et al. Incidence of asthma in adults-report from the Obstructive Lung Disease in Northern Sweden Study. Allergy 1997; 52: 1071-1078.

13. Krzyzanowski M, Lebowitz MD. Changes in chronic respiratory symptoms in two populations of adults studied longitudinally over 13 years. Eur Respir $J$ 1992; 5: 12-20.

14. Huhti E, Ikkala J. A 10-year follow-up study of respiratory symptoms and ventilatory function in a middle-aged rural population. Eur J Respir Dis 1980; 61: 33-45.

15. Huovinen E, Kaprio J, Laitinen LA, Koskenvuo M. Incidence and prevalence of asthma among adult Finnish men and women of the Finnish Twin Cohort from 1975 to 1990, and their relation to hay fever and chronic bronchitis. Chest 1999; 115: 928-936.

16. Bakke P, Gulsvik A, Lilleng P, et al. Postal survey on airborne occupational exposure and respiratory disorders in Norway: causes and consequences of non-response. J Epidemiol Community Health 1990; 44: 316-320.

17. Gulsvik A, Bakke P, Humerfelt S, Omenaas E, Baste V. Measurements of respiratory symptoms and sample size to detect a given difference between treatment groups in obstructive lung disease. Eur Respir Rev 1991; 1: 436-443.

18. Brogger JC, Bakke PS, Gulsvik A. Comparison of respiratory symptoms questionnaires. Int $J$ Tuberc Lung Dis 2000; 4: 83-90.
19. Foss OP, Lund-Larsen PG. Serum thiocyanate and smoking: interpretation of serum thiocyanate levels obtained in a large health study. Scand J Clin Invest 1986; 46: 245-251.

20. Rothman KJ, Greenland S. Measures of disease frequency. In: Rothman $\mathrm{KJ}$, Greenland $\mathrm{S}$, eds. Modern Epidemiology. Philadelphia, LippincottRaven, 1998; pp. 29-46

21. Eagan TM, Bakke PS, Eide GE, Gulsvik A. Predictors and consequences of nonresponse in an 11 years cohort study. Eur Respir J 2000; 16: Suppl. 31, 474s.

22. Upton MN, McConnachie A, McSharry C, et al. Intergenerational 20 year trends in the prevalence of asthma and hay fever in adults: the Midspan family study surveys of parents and offspring. BMJ 2000; 321: 88-92.

23. Hansen EF, Rappeport Y, Vestbo J, Lange P. Increase in prevalence and severity of asthma in young adults in Copenhagen. Thorax 2000; 55: 833-836.

24. Neukirch F, Pin I, Knani J, et al. Prevalence of asthma and asthma-like symptoms in three French cities. Respir Med 1995; 89: 685-692.

25. Manfreda J, Becker AB, Wang PZ, Roos LL, Anthonisen NR. Trends in physician-diagnosed asthma prevalence in Manitoba between 1980 and 1990. Chest 1993; 103: 151-157.

26. Becklake MR, Kauffmann F. Gender differences in airway behaviour over the human life span. Thorax 1999; 54: 1119-1138.

27. Kauffmann F, Becklake M. Maladies obstructives pulmonaires: un paradigme de la complexite des differences de sante entre hommes et femmes. La sante des femmes. Paris, Flammarion, Medecine et Sciences, 1996; pp. 209-233

28. Fujimura M, Sakamoto S, Kamio Y, Matsuda T. Sex difference in the inhaled tartaric acid cough threshold in nonatopic healthy subjects. Thorax 1990; 45: 633-634.

29. Dicpinigaitis PV, Rauf K. The influence of gender on cough reflex sensitivity. Chest 1998; 113: 13191321. 